\title{
Outcome stability of orthopaedic treatment of Skeletal Class III Malocclusion: A study of prognostic agreement between three models
}

\author{
Perinetti, Giuseppe ${ }^{1}$; Bertoli, Alessandra ${ }^{1}$; Dzemidzic, Vildana ${ }^{2}$; Nakaš, Enita ${ }^{2}$; Contardo, Luca ${ }^{1}$ \\ 1 Department of Medical, Surgical and Health Sciences, University of Trieste, Italy \\ 2 Department of Orthodontics, School of Dental Medicine, University of Sarajevo, Bosnia and Herzegovina
}

\begin{abstract}
Introduction: Long-term stability of the treatment outcome of skeletal Class III malocclusion is always not achievable, therefore several prediction models of stability of orthopaedic treatment of the Class III malocclusion have been proposed.

Aim: This cross-sectional study was aimed at the evaluation of the prognostic agreement and association between three different prediction models based on skeletal parameters recorded on lateral cephalograms.

Materials and methods: A total of 75 subjects (34 females and 41 males, age range 7-11, mean age 9.1 \pm 1.2 years) having skeletal Class III malocclusion were included in the study. Prediction models reported by Baccetti et al. 2004 (prediction model 1), Moon et al. 2005 (prediction model 2) and Yoshida et al. 2006 (prediction model 3), were considered. Percentage agreement and unweighted kappa coefficient evaluated the agreement between the prediction models in terms of stable or unstable cases. Multiple regressions were run to evaluate the association between the absolute scores obtained from each of the prediction model (prediction scores).

Results: Percentage agreement ranged from 77.3\% (models 2 and 3) to 89.3\%, (models 1 and 2) and corresponding unweighted kappa coefficients ranged from 0.099 (models 1 and 3) to 0.205 (models 2 and 3). The prediction score from model 3 was significantly associated with those from both the other models; on the contrary, prediction scores from models 1 and 2 were not significantly associated.
\end{abstract}

Conclusions: Aagreement in terms of stability or instability outcome derived from these models is only partial and not satisfactory, even though significant association between the prediction scores denotes a common biological meaning of the models.

Perinetti G, Bertoli A, Dzemidzic V, Nakas E, Contardo L. Outcome stability of orthopaedic treatment of Skeletal Class III Malocclusion: A study of prognostic agreement between three models. South Eur J Orthod Dentofac Res. 2017;4(2):20-25.

Submitted: July 23, 2017; Revised: August 31, 2017; Published: October 14, 2017

\section{INTRODUCTION}

When dealing with orthopaedic treatment of skeletal Class III malocclusion, a long-term stability of the treatment outcome is not always achievable. Although, clinical investigations ${ }^{1-4}$ have reported that orthopaedic treatments are effective to reduce skeletal Class III malocclusion avoiding surgery, there is a tendency for some patients toward re-emergence of the Class III growth pattern, especially during the pubertal growth phase. ${ }^{5}$

Corresponding Author:

Perinetti Giuseppe

Struttura Complessa di Clinica Odontoiatrica e Stomatologica,

Ospedale Maggiore,

Piazza Ospitale 1, 34129 Trieste, Italy

e-mail: G.Perinetti@fmc.units.it
Thus, previous investigations ${ }^{6-10}$ were aided at the identification of individual pre-treatment cephalometric parameters to be used in combination into a prediction model as predictors of stability of orthopaedic treatment of the Class III malocclusion (for review, see Fudalej et al. ${ }^{11}$ ). The tool used in these long-term longitudinal studies was the discriminant analysis ${ }^{6}$ that, according to pre-established pre-treatment variables, is able to discriminate between stable and unstable Class III treatment outcome. This model uses unstandardized discriminant function coefficients that are calculated for each previously selected variable together with a constant. This calculation leads to an equation that assigns a "mean score" for each of the groups (stable or unstable) being compared. Halfway between these scores is the dividing value (critical score) that establishes to which of the groups a patient belongs. 
Therefore, by applying the prediction model to an individual patient prior to the treatment, an individualised absolute score (prediction score) is retrieved that has to be converted into stable or unstable future treatment outcome according to the relative critical score provided for that model.

However, a noteworthy difference in terms of prediction models has been reported in the previous studies ${ }^{11}$ with no evidence of prognostic agreement among the proposed models. Such a lack of evidence implies the choice of the prediction model to be empirical. Therefore, the present cross-sectional study was thus aimed at the evaluation of the prognostic agreement and association between different prediction models based on skeletal cephalometric parameters. The null hypothesis was that no difference in terms of prognostic outcome was seen among the models. In case of agreement, the choice of the prediction model to use might be based on easiness of landmark identification of the lateral cephalogram.

\section{MATERIALS AND METHODS}

\section{Subjects and study design}

The databases between May 2010 and July 2016 of Department of Orthodontics of the Dental Hospital at the University of Sarajevo (Bosnia and Herzegovina) and databases between January 2007 and July 2016 of the Department of Orthodontics of the Dental Hospital at the University of Trieste (Italy) were screened. Accordingly, an initial sample of 149 Caucasian subjects seeking orthodontic treatment and who had never been treated before, was considered for inclusion. As a routine procedure, a signed informed consent for releasing diagnostic records for scientific purposes was obtained from the parents of the subjects prior to entry into the treatment. Procedures followed adhered to the World Medical Organization Declaration of Helsinki ${ }^{12}$ and the protocol was reviewed and approved by the local Ethical Committee. Other inclusion criteria were: i) age between 7 and 11 years old; ii) skeletal Class III malocclusion defined as having anterior crossbite or edge-to-edge incisal relationship and a Wits appraisal $\leq 1.5 \mathrm{~mm}$ and an ANB Angle $\leq 0^{\circ}$ or $\mathrm{ANB}>0^{\circ}$ and $<1^{\circ}$ and a Wits appraisal $\leq-1.5$; iii) being in the pre-pubertal grow phase according to the cervical vertebral maturation method ${ }^{13}$ stages 1 or 2 , or according to the middle phalanx maturation method ${ }^{14}$ stage 1 ; iv) lack of congenital deformities such as a cleft lip and palate; v) good general health with no growth or nutritional problems; vi) absence of major craniofacial or dental anomalies; and vii) availability of a good quality head film. All the lateral head films were recorded by the same operator with a dedicated X-ray machine (KODAK 8000C; Eastman Kodak Company). Settings were of $73-77 \mathrm{kV}, 12 \mathrm{~mA}$ with an exposure time of 0.80 seconds.

After selection, 75 subjects ( 34 females and 41 males, age range $7-11$, mean age $9.1 \pm 1$.2years) were included in the study.

\section{Prediction models}

Articles were identified through a literature survey carried out through the PubMed and SCOPUS databases. The survey covered the period from inceptions to the last access on August 15, 2017. The main used algorithm was as follows: "Class III AND (prediction/predictive OR discriminant OR stability OR relapse OR prognosis/prognostic)". The studies retrieved had to be either RCTs or prospective or retrospective CCTs. They had to include patients with a skeletal Class III malocclusion treated during the pre-pubertal growth phase and with a followup during the post-pubertal phase or adult age of at least 5 years. These studies had to investigate the skeletal stability of the orthopaedic treatment and to identify through a discriminant analysis any skeletal cephalometric parameter/s (and corresponding formula) able to discriminate between a stable and unstable treatment outcome case. No restriction was applied to the type of prognostic parameters collected, as long as they were skeletal parameter. Also, no restrictions were set regarding the type of orthopaedic treatment (facemask or chincap) or whether such treatment was executed once or twice in the same patient, irrespective of a subsequent full fixed appliance treatment. Studies were excluded if information provided were not complete (even after contacting the Authors) or extraction cases were included in the examined samples. A total of three studies (accounting for three models) from Baccetti et al..$^{15}$ (prediction model 1), Moon et al. ${ }^{16}$ (prediction model 2) and Yoshida et al. ${ }^{17}$ (prediction model 3), were included. For data extraction, the following items were considered for each study: sample size and sex distribution, ethnicity, age, main definition of Class III malocclusion, treatment (as rapid maxillary expansion [RME], facemask, chincap, full fixed appliance), length of the follow-up, definition of stable case, percantage of stable cases in the long term, prediction model, critical scores and overall prediction of the model. Details of each study and prediction models are summarised in Table 1 and shown in Figure 1.

Figure 1. Cephalometric parameters used as predictors for outcome stability in functional treatment for Class III malocclusion.

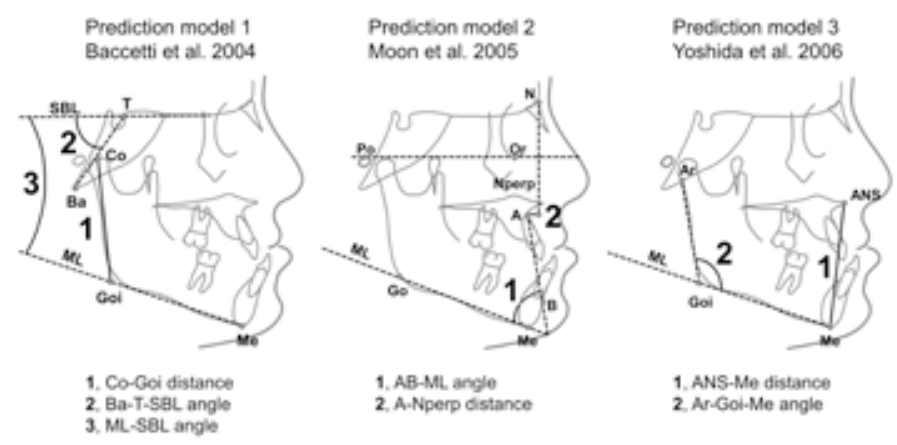

Points: Co, Condylion; Ba, Basion; Goi, Gonial intersection; Me, Menton; Po, Pogonion; $\boldsymbol{N}$, Nasion; Or, Orbitale; Go, Gonion; Ar, Articulare; ANS, Anterior nasal spine. Lines: SBL, Stable basal line; $M L$, Mandibular line; $N$ perp, $N$ perpendicular line. See text further details. 
Table 1. Main features of the previous studies on the cephalometric predictors for outcome stability of functional treatment for Class III malocclusion.

\begin{tabular}{|c|c|c|c|c|c|c|c|c|}
\hline Study & Sample (Ethnicity) & Age (yrs) & $\begin{array}{l}\text { Main definition of } \\
\text { Class III }\end{array}$ & Treatment & $\begin{array}{l}\text { Follow- up } \\
\text { (yrs) }\end{array}$ & Definition of stable case & Prediction model & $\begin{array}{l}\text { Critical score and } \\
\text { overall prediction } \\
\text { of the model }\end{array}$ \\
\hline Baccetti et al. ${ }^{15}$ & $\begin{array}{l}22 \mathrm{~F} ; 20 \mathrm{M} \\
\text { (Caucasians) }\end{array}$ & $8.5 \pm 2.0$ & $\begin{array}{l}\text { - Edge-to-edge } \\
\text { incisal relationship } \\
\text { (at least); } \\
\text { - Wits } \leq 1.5 \mathrm{~mm}\end{array}$ & $\begin{array}{l}\text { RME and FM } \\
\text { followed by FFA }\end{array}$ & -6.5 & $\begin{array}{l}\text { Absence of Class III } \\
\text { molar } \\
\text { relationship and negative } \\
\text { overjet } \\
\text { Successful cases, } 71.4 \%\end{array}$ & $\begin{array}{l}\text { Score }=0.282 \cdot \mathrm{Co}- \\
\text { Goi distance }+ \\
0.205 \bullet B a-T-S B L \\
\text { angle }+0.12 \bullet \mathrm{ML}- \\
\text { SBL angle }-29.784\end{array}$ & $\begin{array}{l}\text { Stable case when } \\
<0.406 \\
\text { Prediction, } 83.3 \%\end{array}$ \\
\hline Moon et al. ${ }^{16}$ & $\begin{array}{l}29 \mathrm{~F} ; 16 \mathrm{M} \\
\text { (Asians) }\end{array}$ & $8.6 \pm 1.5$ & $\begin{array}{l}\text { Anterior crossbite } \\
\text { in either primary } \\
\text { or mixed dentition }\end{array}$ & $\begin{array}{l}\text { Chincap followed } \\
\text { by FFA }\end{array}$ & -8.8 & $\begin{array}{l}\text { - Overbite }>1.5 \mathrm{~mm} \\
\text { and overjet }>2.0 \mathrm{~mm} \\
\text { (stable occlusal status } \\
\text { group) } \\
\text { - With a relapse } \\
\text { tendency to anterior } \\
\text { crossbite but without } \\
\text { indication to surgery } \\
\text { (fair occlusal stability } \\
\text { group) } \\
\text { Successful cases, } 66.6 \%\end{array}$ & $\begin{array}{l}\text { Score }=0.290 \bullet A B- \\
\text { ML angle }-0.501 \\
\text { A-Nperp distance } \\
-18.349\end{array}$ & $\begin{array}{l}\text { Stable case when } \\
>-0.904 \text { (merging } \\
\text { stable occlusal status } \\
\text { and fair occlusal } \\
\text { stability groups) } \\
\text { Prediction, } 77.8 \%\end{array}$ \\
\hline Yoshida et al. ${ }^{17}$ & $\begin{array}{l}32 \mathrm{~F} \\
\text { (Japanese) }\end{array}$ & 10.2 & Anterior crossbite & $\begin{array}{l}\text { Combined FM and } \\
\text { chincap followed } \\
\text { by FFA }\end{array}$ & 5.4 & $\begin{array}{l}\text { Absence of Class III } \\
\text { molar and canine } \\
\text { relationship and negative } \\
\text { overjet } \\
\text { Successful cases, } 50 \%\end{array}$ & $\begin{array}{l}\text { Score }=-0.197 \bullet \text { ANS- } \\
\text { Me distance } \\
-0.125 \bullet \text { Ar-Goi-Me } \\
\text { angle }+28.5\end{array}$ & $\begin{array}{l}\text { Stable case when } \\
>-0.098 \\
\text { Prediction, } 84.4 \%\end{array}$ \\
\hline
\end{tabular}

$\boldsymbol{F}$, females; $\boldsymbol{M}$, males; $\boldsymbol{R} \boldsymbol{M E}$, rapid maxillary expansion; $\boldsymbol{F M}$, protraction face-mask; $\boldsymbol{F F A}$, full fixed appliance. See Figure 1 for details of the cephalometric parameters.

\section{Method error}

With the aim of quantifying the full method error of the recordings for each cephalometric parameter, the method of moments variance estimator was used. This variance estimator has the advantages of not being affected by any unknown bias, i.e. systematic errors, between pairs of measurements. ${ }^{18}$ This analysis was performed on 20 pairs of recordings randomly selected, and was calculated for each cephalometric parameter as mean and $95 \%$ confidence interval (CI) calculated as previously reported. ${ }^{19}$

\section{Data analysis}

To determine the degree of agreement between the three prediction models, the frequencies of predicted stable and unstable cases were crosstabulated, and percentages of agreement along with 95\% CIs between the three prediction models were calculated. Subsequently, non-weighted kappa coefficients ${ }^{18}$ along with 95\% CIs were also calculated between the three prediction models. The kappa coefficient ranges from zero for no agreement to 1 for perfect agreement, and the following standards for strength of agreement for the kappa coefficient have proposed: 0.01-0.20, slight; 0.21-0.40, fair; 0.41-0.60, moderate; 0.61-0.80, substantial; and $>0.80$ almost perfect.

Moreover, the association between the prediction scores (i.e. the score derived from each model) were investigated by means of backward multiple linear regressions. In particular, age and sex were entered in each model having the scores from a prediction model as the dependent variable and the scores from the other two models as independent variables. Thus, three different multiple linear regressions were run each for any of the prediction models. The cut-off levels of significance used were 0.01 and 0.05 for entry and removal, respectively. For each multiple regression model, multi-co-linearity among the remaining explanatory variables was also again checked for though the tolerance and variance inflation factor parameters.

The Statistical Package for Social Sciences software 20.0 (SPSS ${ }^{\bullet}$ Inc., Chicago, Illinois, USA) and MedCalc ${ }^{\oplus}$ software 12.3.3.0 (MedCalc Software, Mariakerke, Belgium) were used to perform the statistical analyses and, where not differently specified, data were presented as mean \pm standard deviation. A p value less than 0.05 was considered as significant.

\section{RESULTS}

For the linear and angular measurements, greatest method errors were $0.9 \mathrm{~mm}(0.7-1.3)$ and $1.2^{\circ}(0.9-1.7)$, for the ANS$\mathrm{Me}$ and Ar-Goi-Me angle, respectively.

The cephalometric features of the whole sample as mean $\pm S D$ were as follows: the SNA, SNB and ANB angles were $78.7^{\circ} \pm 3.4,80.1^{\circ}$ \pm 3.0 and $-1.4^{\circ} \pm 1.9$, respectively; the FOP Wits was of $-4.9 \mathrm{~mm}$ \pm 2.6 ; the $\mathrm{SN}-\mathrm{GoGn}$ angle was $32.8^{\circ} \pm 4.4$; inclinations of upper incisors on maxillary plane and of lower Incisor on mandibular plane were $108.7^{\circ} \pm 7.6$ and $85.1^{\circ} \pm 8.0$, respectively; and overjet and overbite were $-0.5 \mathrm{~mm} \pm 2.3$ and $1.0 \mathrm{~mm} \pm 2.5$, respectively. 
Results on the cephalometric scores of each of the prediction variables included in the three models are summarised in Table 2. For prediction model 1, Co-Goi distance, Ba-T-SBL angle and ML-SBL angle were $47.7 \mathrm{~mm} \pm 4.4,56.6^{\circ} \pm 5.2$ and $26.3^{\circ} \pm 4.8$, respectively, while the overall prediction score was $-1.6 \pm 1.4$. For prediction model 2, AB-ML angle and A-Nperp distance, were $63.1^{\circ} \pm 4.8$ and $-3.4 \mathrm{~mm} \pm 2.6$, respectively, while the overall prediction score was $1.7 \pm 1.6$. For prediction model 3, ANS-Me distance and Ar-Goi-Me angle, were $58.0 \mathrm{~mm} \pm 4.9$ and $128.9^{\circ}$ \pm 6.7 , respectively, while the overall prediction score was $1.0 \pm 1$. 3 .

Results on the agreement between the three prediction models in terms of predicted stable and unstable cases are summarised in Table 3. The number of stable cases retrieved were 70 (93.3\%) for both the prediction models 1 and 2, and 59 (78.7\%) for the prediction model 3. The overall percentages of agreement ranged from $77.3 \%$ and $89.3 \%$ between prediction models 2 and 3 and prediction models 1 and 2, respectively. The unweighted kappa coefficients were all very low with the highest value of 0.205 (between prediction models 1 and 2), and none was statistically significant.

Table 2. Cephalometric scores of each of the variables included in the three prediction models $(n=75)$.

\begin{tabular}{|c|c|c|c|}
\hline Prediction model/Parameter & Mean \pm SD & Minimum & Maximum \\
\hline \multicolumn{4}{|l|}{ Model 1 (Baccetti et al. ${ }^{15}$ ) } \\
\hline Co-Goi distance (mm) & $47.7 \pm 4.4$ & 39.6 & 57.5 \\
\hline Ba-T-SBL angle $\left({ }^{\circ}\right)$ & $56.6 \pm 5.2$ & 43.2 & 67.4 \\
\hline ML-SBL angle $\left({ }^{\circ}\right)$ & $26.3 \pm 4.8$ & 12.4 & 37.8 \\
\hline Prediction score & $-1.6 \pm 1.4$ & -4.2 & 1.5 \\
\hline \multicolumn{4}{|l|}{ Model 2 (Moon et al. $\left.{ }^{16}\right)$} \\
\hline AB-ML angle $\left({ }^{\circ}\right)$ & $63.1 \pm 4.8$ & 50.4 & 71.7 \\
\hline A-Nperp distance (mm) & $-3.4 \pm 2.6$ & -9.9 & 1.3 \\
\hline Prediction score & $1.7 \pm 1.6$ & -2.7 & 5.2 \\
\hline \multicolumn{4}{|l|}{ Model 3 (Yoshida et al. ${ }^{17}$ ) } \\
\hline ANS-Me distance (mm) & $58.0 \pm 4.9$ & 47.5 & 70.8 \\
\hline Ar-Goi-Me angle $\left({ }^{\circ}\right)$ & $128.9 \pm 6.7$ & 112.0 & 143.8 \\
\hline Prediction score & $1.0 \pm 1.3$ & -3.4 & 3.5 \\
\hline
\end{tabular}

Results of the backward multiple linear regression with each of the three prediction scores as dependent variables are summarised in Table 4. Age and sex did not show any significant association with any of the three prediction models. When scores form prediction model 1 was considered as the dependent variable, only model 3 showed a significant negative association $(B,-0.475, P=0.000)$ with a $\mathrm{R} 2$ of 0.181 ; when score from prediction model 2 was considered as the dependent variable, only model 3 showed a significant positive association ( $(3,0.560$, $\mathrm{P}=0.000$ ) with a $\mathrm{R} 2$ of 0.206 ; when score form prediction model 3 was considered as the dependent variable, both models 1 and 2 showed a significant negative association $(B,-0.369, P=0.001$ and $B,-0.346, P=0.000$ ) with a $R 2$ of 0.363 .
Table 3. Agreement between the three prediction models in terms of predicted stable and unstable cases $(n=75)$.

\begin{tabular}{|c|c|c|c|c|c|}
\hline \multirow{2}{*}{$\begin{array}{l}\text { Prediction } \\
\text { model }\end{array}$} & \multirow{2}{*}{ Outcome } & \multicolumn{2}{|c|}{ Model 2 (Mon et al. $\left.{ }^{16}\right)$} & \multicolumn{2}{|c|}{ Model 3 (Yoshida et al..$^{17}$ ) } \\
\hline & & Stable & Unstable & Stable & Unstable \\
\hline \multirow{4}{*}{$\begin{array}{l}\text { Model } 1 \\
\text { (Baccetti et al. }{ }^{15} \text { ) }\end{array}$} & Stable & 66 & 4 & 54 & 13 \\
\hline & Unstable & 4 & 1 & 2 & 3 \\
\hline & Overall agreement & \multicolumn{2}{|c|}{$89.3 \%(80.1-95.3)$} & \multicolumn{2}{|c|}{$80.0 \%(62.9-88.4)$} \\
\hline & Kappa coefficient & \multicolumn{2}{|c|}{$0.143(0-0.490)$} & \multicolumn{2}{|c|}{$0.205(0-0.454)$} \\
\hline \multirow{4}{*}{$\begin{array}{l}\text { Model } 2 \\
\left(\text { Moon et al. }{ }^{16} \text { ) }\right.\end{array}$} & Stable & - & - & 56 & 14 \\
\hline & Unstable & - & - & 3 & 2 \\
\hline & Overall agreement & \multicolumn{4}{|c|}{$77.3 \%(66.2-86.2)$} \\
\hline & Kappa coefficient & \multicolumn{2}{|c|}{ - } & \multicolumn{2}{|c|}{$0.099(0-0.324)$} \\
\hline
\end{tabular}

Overall agreements and unweighted kappa coefficient are presented as mean (95\% confidence interval).

Table 4. Results of the backward multiple linear regressions with each of the three prediction scores as dependent variable $(n=75)$.

\begin{tabular}{llcc}
\hline Dependent variable & $\mathbf{B}(\mathbf{S E})$ & $\mathbf{t}$ & P value \\
\hline Prediction model 1, R2= $\mathbf{0 . 1 8 1}$ & & & \\
\hline Prediction model 3 & $-0.475(0.114)$ & 4.023 & $\mathbf{0 . 0 0 0}$ \\
\hline Prediction model 2, R2= $\mathbf{0 . 2 0 6}$ & & & \\
\hline Prediction model 3 & $0.560(0.129)$ & 4.353 & $\mathbf{0 . 0 0 0}$ \\
\hline Prediction model 3, R2= $\mathbf{0 . 3 6 3}$ & & & \\
\hline Prediction model 1 & $-0.369(0.088)$ & 4.204 & $\mathbf{0 . 0 0 1}$ \\
\hline Prediction model 2 & $-0.346(0.077)$ & 4.552 & $\mathbf{0 . 0 0 0}$ \\
\hline
\end{tabular}

Age and sex also entered in each model yielded no significant associations. ß, coefficient of correlation, $\boldsymbol{S E}$, standard error.

\section{DISCUSSION}

The present study analysed the prognostic agreement and association between three prediction models for the longterm outcome of orthopaedic treatment of skeletal Class III malocclusion delivered in pre-pubertal patients. A crosssectional analysis on 75 Class III subjects has been followed to retrieve the treatment outcome according to each of the three prediction models. Although the models showed over $70 \%$ of agreement, little degree of association were seen only for the model 3 with the other two models.

When dealing with a skeletal Class III malocclusion in a prepubertal patient, a reliable prediction model may provide a valuable tool. Indeed, if instability of the orthopaedic treatment outcome is expected, intervention might be delayed until completion of growth. The prediction models considered in the present investigations showed very high predictability with values ranging from $77.8 \%{ }^{16}$ to $84.4 \%{ }^{17}$ and these were 
selected on the basis of long-term monitoring beginning at the pre-pubertal and ending at the post-pubertal growth phase lasting $\sim 5.4^{17}$ to $\sim 8.8$ years $^{16}$ (Table 1 ). However, in spite of the attempt to retrieve similar studies, these three investigations included patients of different ethnicity as Baccetti et al. ${ }^{15}$ reported on a group of Caucasians, Moon et al. ${ }^{16}$ included Asian patients and Yoshida et al. ${ }^{17}$ treated only Japanese girls. These studies also used different treatment modalities as Baccetti et al. ${ }^{15}$ used RME and facemask followed by full fixed appliance, Moon et al. ${ }^{16}$ used chincap followed by full fixed appliance and Yoshida et al. ${ }^{17}$ used facemask and chincap followed by full fixed appliance. Therefore, conclusions have to take into account possible differences in the ethnicity and treatment modalities herein considered.

Other excluded studies reported on prediction models related to the orthopaedic treatment of skeletal Class III malocclusion. For instance, the study by Tahmina et al. ${ }^{9}$ was excluded since the normalized $\mathrm{z}$ score (calculated according to a Japanese control group) were used instead the actual values. The study by Battagel $^{6}$ was excluded because incorporated soft tissue parameters in the prediction model being thus significant different that parameters used in the other investigations. The study by Wells et al. ${ }^{10}$ was not included because the lack of data regarding the sample description. The study by Schuster et al. ${ }^{8}$ was not included because the proposed model relied on the occlusal plane. Finally, the most recent studies by Ghiz et al. ${ }^{7}$ and Choi et al. ${ }^{20}$ were excluded because a multiple logistic regression was used instead of a discriminant analysis.

A previous systematic review ${ }^{11}$ concluded that a universal predictor of outcome of orthopaedic treatment of skeletal Class III malocclusion may be questionable. Such a conclusion was based on the evidence that different studies reported different models, lacking an analysis on the agreement between prediction models. Moreover, the review ${ }^{11}$ was not restricted to investigations using discriminant analysis.

All the prediction models analysed included both angular and linear cephalometric parameter (Figure 1). Therefore, proper magnification factor has to be applied to ensure reliable outcomes. These models yield absolute values from which nominal (dichotomous) treatment outcome (i.e. stable or unstable) is derived according to a critical score (Table 1). Although the previous study by Choi et al. ${ }^{20}$ evaluated the concordance between several prediction models, it was limited to the nominal treatment outcome. However, the absolute values retrieved by the models is also worth of analysis since nominal outcomes may also show poor agreement in combination with association of the absolute scales from which they are derived (see Results). Herein, both the nominal outcomes (either stable or unstable) and scores from which they were derived have been analysed for the first time.
The present results showed only moderate agreement between the model 3 with the other two models (Tables 3 and 4). Of interest, the treatment related to the prediction model 3 was a combinational use of facemask with chincap ${ }^{17}$, a modality which has common features with the treatments related to the other prediction models 1 and 2 that were either facemask ${ }^{15}$ or chincap ${ }^{16}$, respectively. On the contrary, prediction models 1 and 2 did not show any significant agreement (Table 2) or association (Table 3). It might be hypothesised that the prediction models are related to the treatment that has effects on the maxilla and mandible growth together (i.e. facemask) or on the mandible growth alone (i.e. chincap). Therefore, the model to be used to predict outcome treatment for skeletal Class III malocclusion should take into account the treatment modality. However, agreement among prognostic models does not imply per se prognostic accuracy; it only implies that models yields to similar outcomes and, therefore, a given model may be preferred on the basis of easiness of landmark identification. In this regard, previous investigations ${ }^{11,20}$ reported that stability of the orthopaedic treatment outcome of skeletal Class III malocclusion depends on several factors such treatment modality, timing, outcome evaluation, severity of malocclusion and individual patient responsiveness, making questionable the existence of a universal predictor.

In spite of the difference in the prediction parameters among the three models, ${ }^{15-17}$ very few of these parameters was related to the maxilla or to the sagittal position of the basal bones, such as the A-Nperp distance in the prediction model $2 .{ }^{17}$ Even though the models showed herein little or no agreement (Tables 3), it can be speculated that parameters related to the vertical components (especially mandibular), are likely predictors of the longterm outcome of orthopaedic treatment for skeletal Class III malocclusion. Even in one ${ }^{7}$ of the investigations herein excluded, mandibular parameters were seen to have predictive potential in terms of long-term success of the orthopaedic treatment.

The present results from the multiple linear regressions show that the different prediction models yield to scores that may be associates, irrespective of age and sex (Table 4). This implies that cephalometric variables into each model share some common prediction features. In line with recent evidence, ${ }^{20}$ when the scores of the prediction models are converted into either stable or unstable cases, the concordance, may be up to 89\% (Table 3). However, when considering the unweighted kappa coefficients, the agreement between the three prediction models was minimal with no statistical significance (Table 3). This apparent inconsistence was due to the high number of stable cases seen in all the models in combination with (relatively) few cases of disagreement. Therefore, the main limitation of the prediction models proposed to date would reside in the critical value according to which the overall score is either recoded into a stable or unstable case. Future investigations 
are warranted to determine whether such critical values may be adjusted to increase reliability or agreement between prediction models. Even though the present study did not include a group of treated subjects followed longitudinally in the long-term, the associations between the prediction models constitutes an indirect prove that several vertical cephalometric variables would be responsible for the long-terms stability of the treatment outcomes, although reference values cannot be set at present.

\section{CONCLUSIONS}

An association between scores of the prediction models was seen, denoting a common biological meaning of the models. However, the agreement in terms of stability or instability outcome derived from these models is only partial and not satisfactory. These results suggest that clinicians need to be cautious when predicting the long-term stability of orthopaedic treatment of skeletal Class III malocclusion, especially when a single prediction model is used. Therefore, patients have to be informed about the re-establishing of a pre-treatment skeletal Class III malocclusion, even when a stable treatment outcome is retrieved according to the models herein analysed.

\section{REFERENCES}

1. Graber LW. Chin cup therapy for mandibular prognathism. Am J Orthod 1977;72:23-41.

2. Baik HS. Clinical results of the maxillary protraction in Korean children. Am J Orthod Dentofacial Orthop 1995;108:583-92.

3. Macdonald KE, Kapust AJ, Turley PK. Cephalometric changes after the correction of class III malocclusion with maxillary expansion/ facemask therapy. Am J Orthod Dentofacial Orthop 1999;116:1324.

4. Toffol LD, Pavoni C, Baccetti T, Franchi L, Cozza P. Orthopedic treatment outcomes in Class III malocclusion. A systematic review. Angle Orthod 2008;78:561-73.

5. Westwood PV, McNamara JA, Jr., Baccetti T, Franchi L, Sarver DM. Long-term effects of Class III treatment with rapid maxillary expansion and facemask therapy followed by fixed appliances. Am J Orthod Dentofacial Orthop 2003;123:306-20.

6. Battagel JM. Discriminant analysis: a model for the prediction of relapse in Class III children treated orthodontically by a nonextraction technique. Eur J Orthod 1993;15:199-209.

7. Ghiz MA, Ngan P, Gunel E. Cephalometric variables to predict future success of early orthopedic Class III treatment. Am J Orthod Dentofacial Orthop 2005;127:301-6.

8. Schuster G, Lux CJ, Stellzig-Eisenhauer A. Children with class III malocclusion: development of multivariate statistical models to predict future need for orthognathic surgery. Angle Orthod 2003;73:136-45.

9. Tahmina K, Tanaka E, Tanne K. Craniofacial morphology in orthodontically treated patients of class III malocclusion with stable and unstable treatment outcomes. Am J Orthod Dentofacial Orthop 2000;117:681-90.

10. Wells AP, Sarver DM, Proffit WR. Long-term efficacy of reverse pull headgear therapy. Angle Orthod 2006;76:915-22.
11. Fudalej P, Dragan M, Wedrychowska-Szulc B. Prediction of the outcome of orthodontic treatment of Class III malocclusions--a systematic review. Eur J Orthod 2011;33:190-7.

12. World Medical Association. WMA Declaration of Helsinki - Ethical Principles for Medical Research Involving Human Subjects; 2008.

13. Baccetti T, Franchi L, McNamara JA, Jr. The cervical vertebral maturation (CVM) method for the assessment of optimal treatment timing in dentofacial orthopedics. Semin Orthod 2005;11:119-29.

14. Perinetti G, Sbardella V, Contardo L. Diagnostic reliability of the third finger middle phalanx maturation (MPM) method in the identification of the mandibular growth peak. Eur J Orthod 2017;39:194-201.

15. Baccetti T, Franchi L, McNamara JA, Jr. Cephalometric variables predicting the long-term success or failure of combined rapid maxillary expansion and facial mask therapy. Am J Orthod Dentofacial Orthop 2004; $126: 16-22$

16. Moon YM, Ahn SJ, Chang YI. Cephalometric predictors of longterm stability in the early treatment of Class III malocclusion. Angle Orthod 2005;75:747-53.

17. Yoshida I, Yamaguchi N, Mizoguchi I. Prediction of post-treatment outcome after combined treatment with maxillary protraction and chincap appliances. Eur J Orthod 2006;28:89-96.

18. Perinetti G. StaTips Part III: Assessment of the repeatability and rater agreement for nominal and ordinal data. South Eur J Orthod Dentofac Res 2017;4:3-4.

19. Springate SD. The effect of sample size and bias on the reliability of estimates of error: a comparative study of Dahlberg's formula. Eur J Orthod 2012;34:158-63

20. Choi YJ, Chang JE, Chung CJ, Tahk JH, Kim KH. Prediction of long-term success of orthopedic treatment in skeletal Class III malocclusions. Am J Orthod Dentofacial Orthop 2017;152:193-203. 